\title{
False-negative results using Neisseria gonorrhoeae porA pseudogene PCR - a clinical gonococcal isolate with an N. meningitidis porA sequence, Australia, March 2011
}

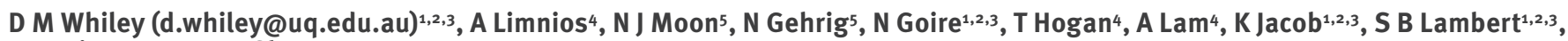
M D Nissen $1,2,3,5, T$ P Sloots $1,2,3,5$

1. Queensland Paediatric Infectious Diseases Laboratory, Queensland Children's Health Services, Queensland, Australia

2. Queensland Children's Medical Research Institute, The University of Queensland, Queensland, Australia

3. Clinical Medical Virology Centre, Sir Albert Sakzewski Virus Research Centre, The University of Queensland, Queensland, Australia

4. World Health Organization Collaborating Centre for Sexually Transmitted Diseases, Sydney, Microbiology Department, South Eastern Area Laboratory Services, The Prince of Wales Hospital, Sydney, New South Wales, Australia

5. Microbiology Department, Hunter Area Pathology Service, Division of Pathology North, Newcastle, New South Wales, Australia

Citation style for this article:

Whiley DM, Limnios A, Moon NJ, Gehrig N, Goire N, Hogan T, Lam A, Jacob K, Lambert SB, Nissen MD, Sloots TP. False-negative results using Neisseria gonorrhoeae porA pseudogene PCR - a clinical gonococcal isolate with an N. meningitidis porA sequence, Australia, March 2011.

Euro Surveill. 2011;16(21):pii=19874. Available online: http://www.eurosurveillance.org/ViewArticle.aspx?Articleld=19874

Article published on 26 May 2011

The gonococcal porA pseudogene is a popular target for in-house Neisseria gonorrhoeae PCR methods. With this study we present two novel findings: the first case of an $N$. gonorrhoeae porA pseudogene PCR false-negative result caused by sequence variation, and in the same organism, the first description of a clinical N. gonorrhoeae strain harbouring an N. meningitidis porA sequence.

In this report, we describe the first case of a Neisseria gonorrhoeae false-negative test result using an $N$. gonorrhoeae porA pseudogene PCR method, caused by sequence variation. Nucleic acid amplification tests (NAATs) are widely used for the detection of gonorrhoea, yet there are challenges for $N$. gonorrhoeae NAATs because of the considerable sequence variation and genetic exchange that is exhibited by the Neisseria genus. Many gonococcal NAATs are known to crossreact with commensal Neisseria strains necessitating the use of supplementary testing $[1,2]$. In addition, sequence-related false-negative results have also been reported for NAATs targeting certain gonococcal sequences. These include the $N$. gonorrhoeae $с p p B$ and opa genes $[3,4]$.

In March 2011, a young man in his early 20 s presented with anal pain to a sexual health clinic in Newcastle, New South Wales, Australia. The man reported having recently had numerous sexual contacts with men (MSM), some with overseas visitors, including from the United States, but reported no recent overseas travel. Pharyngeal and rectal swabs, as well as a urine sample were obtained and submitted for $N$. gonorrhoeae testing. The urine sample and rectal swab were tested by NAAT, and both swab samples were tested by bacterial culture. A summary of results is provided in the Table.

The rectal swab provided positive results for $N$. gonorrhoeae by Cobas4800 CT/NG testing (Roche Diagnostics, Australia) which targets a direct repeat

\section{TABLE}

Culture and NAAT results for Neisseria gonorrhoeae by anatomical site and type of sample, New South Wales, Australia, March 2011

\begin{tabular}{|l|c|c|c|c|}
\hline \multirow{2}{*}{$\begin{array}{l}\text { Anatomical site / } \\
\text { type of sample }\end{array}$} & Culture & Neisseria gonorrhoeae diagnostic methods & $\begin{array}{c}\text { LightCycler PCR } \\
\text { (porA pseudogene) }\end{array}$ & $\begin{array}{c}\text { TaqMan PCR } \\
\text { (porA pseudogene) }\end{array}$ \\
\cline { 2 - 5 } & NP & Negative & NP & NP \\
\hline Urine sample & Positive & Positive & Negative & Negative \\
\hline Rectal swab & NA & Positive & Negative & Negative \\
\hline Rectal isolate & Positive & NP & NP & NP \\
\hline Pharyngeal swab & NA & Positive & Negative & Negative \\
\hline Pharyngeal isolate & & &
\end{tabular}

NA: not applicable; NAAT: nucleic acid amplification test; NP: not performed. 
region, DR-9, with a cycle threshold value of 27 , and $N$. gonorrhoeae was subsequently isolated from both the pharyngeal and rectal swabs. The urine sample was negative by NAAT (Cobas4800). Following Australian public health laboratory network guidelines [2] which require supplementary testing for $N$. gonorrhoeae NAAT-positive samples, a DNA extract from the rectal sample was tested using a LightCycler-hybridisation probe-based PCR protocol targeting the gonococcal porA pseudogene [5]. The porA pseudogene is a target widely used for this purpose and has previously been shown to be highly conserved and specific to $N$. gonorrhoeae [5-8]. Negative results were obtained using the LightCycler method for both the DNA extract of the rectal swab as well as the clinical isolates cultured from the pharyngeal and rectal sites. When the rectal sample and the rectal and pharyngeal isolates were subsequently tested using a TaqMan-based $N$. gonorrhoeae porA pseudogene (porA-monoplex) assay, the results were also negative [8]. Testing of the clinical isolates using the Cobas $4800 \mathrm{CT} / \mathrm{NG}$ assay provided positive results for $N$. gonorrhoeae, with cycle threshold values of 17 for both isolates.

The clinical isolates were further characterised phenotypically and genotypically. Both isolates were indistinguishable and were identified phenotypically [9] as N. gonorrhoeae by Gram stain, colonial morphology on modified New York City agar, oxidase, superoxol and rapid carbohydrate utilisation tests. The isolates were tested for prolyliminopeptidase (PIP) activity, auxotyped, serogrouped and the serovar determined by coagglutination reactions with 14 monoclonal reagents (Boule, Huddinge, Sweden). Both isolates tested positive for PIP, were prototrophs and belonged to a common serovar, 'Bropyst'. An identification of N. gonorrhoeae was also provided by the Bruker Biotyper matrix-assisted laser desorption ionisation time of

\section{FIGURE}

Sequence alignment of porA sequences of Neisseria gonorrhoeae FA1090 strain ${ }^{\mathrm{a}}, N$. gonorhoeae porA-variant ${ }^{\mathrm{b}}$ and $N$. meningitidis 278 strain $^{c}$

N.gonorrhoeae (FA1090) N.gonorrhoeae (variant) N.meningitidis (278)

N.gonorrhoeae (FA1090) N.gonorrhoeae (variant) N.meningitidis (278)

N.gonorrhoeae(FA1090) N.gonorrhoeae (variant) N.meningitidis (278)

N.gonorrhoeae(FA1090) N.gonorrhoeae (variant) N.meningitidis ( 278)

N.gonorrhoeae (FA1090) N.gonorrhoeae (variant) N.meningitidis (278)

N.gonorrhoeae (FA1090) N.gonorrhoeae (variant) N.meningitidis (278)

N.gonorrhoeae (FA1090) N.gonorrhoeae (variant) N.meningitidis (278)

N.gonorrhoeae (FA1090) N.gonorrhoeae (variant) N.meningitidis (278)

N.gonorrhoeae (FA1090) N.gonorrhoeae (variant) N.meningitidis (278)

40

60

$70 \quad 80$

90

TTGGCAGCTCGAGCAAGACGTATCCGTTGCCGGCGGCGGGCGACCCGTTGGGGTAACAGGGAATCCTTTATCGGCTTGGCAGGCGAATT

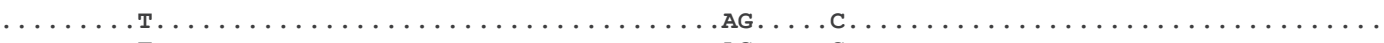

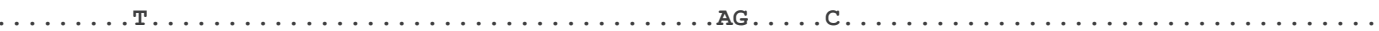

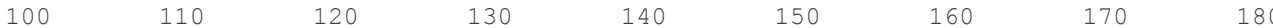

CGGCACGGCGCTCGCCGGTCGCGTTGCGAATCCGTTTGGCGATGCCAGCAAAGCCATTGATCCTTGGGACAGCAATAATAATGTGGCTTC

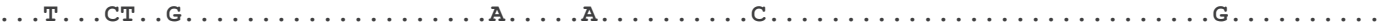

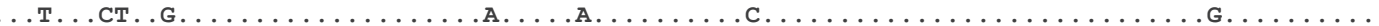
250 $260 \quad 270$ GCAATTGGGTATTTTCAAACGCCACGACGGTATGCCGGTTTCCGTGCGTTACGATTCCCCCGGATTTTCCGGTTTCAGCGGCAGCATTCA

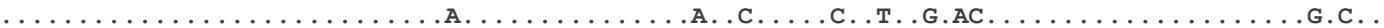

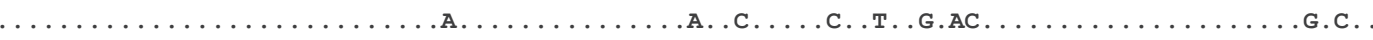

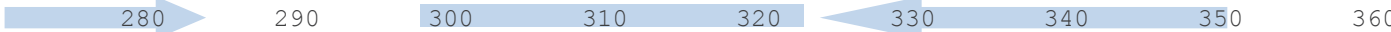
ATTTGTTCCGAGTCAAAACAGCAAGTCCGCCTATACGCCTGCTACTTTCACGCTGGAAAGTAATCAGATGAAACCAGTTCCGGCTGTTGT

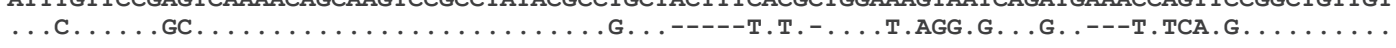

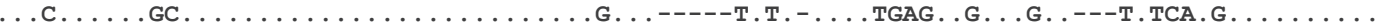

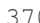

380

$390 \quad 400-410$

420

430

440

450 CGGCAAGCCGGGGTCGGATGTGTATTATGCCGGTCTGAATTACAAAAATGGCGGCTTTTTCGGAAATTATGCCCTTAAATATGCGAAACA

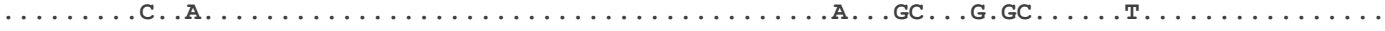

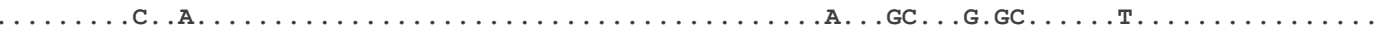
460 $470 \quad 480 \quad 490 \quad 500$ 510 $520 \quad 530$ 540 CGCCAATGAGGGGATGATGCTTTCTTTTTGTTCTTGCTCGGCAGAGCGAGTGAT-_-_-_-_-_-ACCGATCCATTGAAAAACCATCA

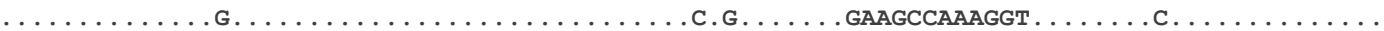

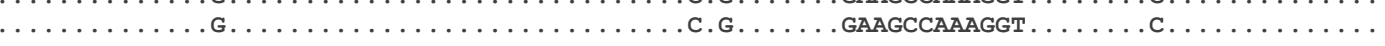
$\begin{array}{llllllll}550 & 560 & 570 & 580 & 590 & 600 & 610 & 620\end{array}$ GGTACACCGCCTGACGGGCGGCTATGGGGAAGGCGGCTTGAATCTCGCCTTGGCGGCTCAGTTGGATTTGTCTGAAAATGCCGACAAAAC

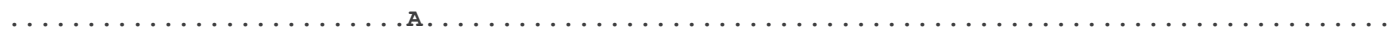

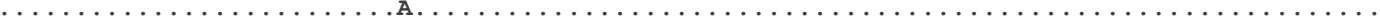
650 670 680 690 710 720 CAAAAACAGTACGACCGAAATTGCCGCCACTGCTTCCTACCGCTTCGGTAATACAGTCCCGCGCATCAGCTATGCCCATGGTTTCGACTT

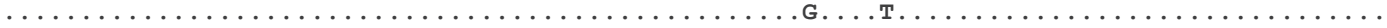

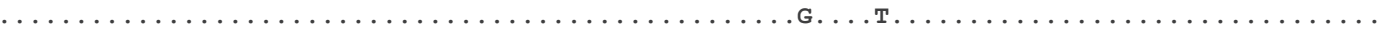

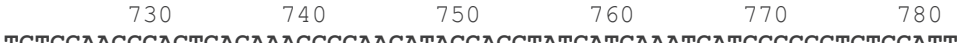

TGTCGAACGCAGTCAGAAACGCGAACATACCAGCTATGATCAAATCATCGCCGGTGTCGATT

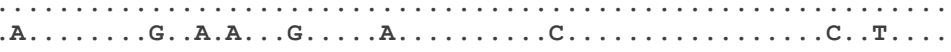

Genbank accession AJ223447.

${ }^{b}$ Pseudogene PCR negative strain from this study.

' Genbank accession GQ173789.

Forward and reverse primer targets of the TaqMan-based Neisseria gonorhoeae porA pseudogene PCR are represented by arrows at positions 262 to 284 and 324 to 250 respectively.

The probe target is represented by the box at position 298 to 323 . 
flight mass spectrophotometer (MALDI TOF MS) Maldi Biotyper (Bruker Biosciences Pty Ltd.). Antimicrobial resistance patterns for these isolates were characteristic of $N$. gonorrhoeae, as determined by the minimum inhibitory concentrations (MICs) using the agar plate incorporation method of the Australian Gonococcal Surveillance Programme and using the CDS Antibiotic Susceptibility criteria [10]. The clinical isolates had chromosomally-mediated penicillin resistance (MIC: $2.0 \mathrm{mg} / \mathrm{L}$ ), quinolone resistance (ciprofloxacin MIC: 16.0 $\mathrm{mg} / \mathrm{L}$ ), decreased susceptibility to ceftriaxone (MIC: $0.03 \mathrm{mg} / \mathrm{L}$ ) and sensitivity to azithromycin and spectinomycin. Both isolates were of multilocus sequence type (MLST) 1901 (abcZ 109, adk 39, aroE 170, fumC 111, gdh 148, pdh (153, pgm 65). This is an N. gonorrhoeae MLST type previously observed in Australia and elsewhere $[11,12]$ and representatives of this type have previously provided positive results by porA-pseudogene PCR in our laboratory (data not shown). Using N. gonorrhoeae multi-antigen sequence typing (NG-MAST), both isolates harboured the previously described POR and tbpB types, 1297 and 983 respectively, which represented a novel NG-MAST type 5377. It should be noted that MLST and NG-MAST are not performed as a routine part of $N$. gonorrhoeae investigations in our laboratory, therefore isolates of these MLST and NG-MAST types may have previously been circulating in our population but not characterised.

To examine the basis of the porA pseudogene PCR false-negative results, a 773 base-pair fragment of the porA sequences of each isolate were amplified using primers CGGCTCGTTTATCGGCTT and GGTATTTCGTTTCAGCCAAGC and subjected to DNA sequencing. The porA-pseudogene PCR-negative $N$. gonorrhoeae strains from this study exhibited only $90 \%$ homology with the reference $N$. gonorrhoeae FA1090 strain (genbank accession number AJ223447) and had multiple mismatches and deletions evident in the primer and probe targets for the porA pseudogene Taqman-based-PCR (Figure) and for the LightCycler hybridisation probe-based method targeting the same region (data not shown).

Notably, genbank blast searching indicated that the porA sequence from the clinical isolates in this study were more similar to that of $N$. meningitidis, having $99 \%$ homology with $N$. meningitidis 278 strain (Genbank accession GQ173789). Only the last 60 bases of the 773 base porA sequence provided greater homology with $N$. gonorrhoeae than with $N$. meningitidis.

\section{Conclusions}

Overall the results show that the rectal and pharyngeal $N$. gonorrhoeae isolates from this patient were typical in terms of genotypic and phenotypic characteristics, except that they had acquired a meningococcal porA sequence presumably through horizontal genetic exchange and recombination. This is yet another example of the problems faced with molecular detection of $N$. gonorrhoeae, and with PCR-based diagnostics more generally. For $N$. gonorrhoeae, the problem is exacerbated by the fact that the species comprises numerous subtypes that exhibit considerable sequence diversity as well as propensity to mutate. Notably, the distribution of subtypes can vary geographically, temporally, and between patient groups. This has implications for the performance of $N$. gonorrhoeae NAATs: firstly, the performance may vary between patient populations because of the presence of different subtypes; but secondly, as in our case, the performance within a given population can suddenly change either due to the importation of new strains or mutation of currently circulating strains. In our opinion, the use of different methods, such as NAAT and bacterial culture in parallel, or multi-target NAAT assays provides the most suitable means of circumventing these problems, and to this extent we have previously described a duplex real-time PCR assay for detecting $N$. gonorrhoeae combining both the porA pseudogene and opa targets [8]. To date, we have not observed any other N. gonorrhoeae isolates with a meningococcal porA sequence in our laboratory and to our best knowledge this has also not been observed elsewhere. Given the propensity for gonococci to spread through populations, we consider it likely that this strain is more widespread. Further investigations including contact tracing and prospectively testing $N$. gonorrhoeae isolates by porA pseudogene $P C R$, are continuing.

\section{Acknowledgments}

We thank Dr Stephen Graves, Lynelle Tilbrook and Brendan Goswell of the Microbiology Department, Hunter Area Pathology Service, Division of Pathology North, for their assistance with this study.

This study was conducted as part of the reference work of the National Neisseria Network, Australia.

\section{References}

1. Johnson RE, Newhall WJ, Papp JR, Knapp JS, Black CM, Gift TL, et al. Screening tests to detect Chlamydia trachomatis and Neisseria gonorrhoeae infections--2002. MMWR Recomm Rep. 2002;18(51):1-38.

2. Smith DW, Tapsall JW, Lum G. Guidelines for the use and interpretation of nucleic acid detection tests for Neisseria gonorrhoeae in Australia: a position paper on behalf of the Public Health Laboratory Network. Commun Dis Intell. 2005;29(4):358-65.

3. Bruisten SM, Noordhoek GT, van den Brule AJ, Duim B, Boel $\mathrm{CH}, \mathrm{El}-\mathrm{Faouzi} \mathrm{K}$, et al. Multicenter validation of the cppB gene as a PCR target for detection of Neisseria gonorrhoeae. J Clin Microbiol. 2004;42(9):4332-4.

4. Geraats-Peters CW, Brouwers M, Schneeberger PM, van der Zanden AG, Bruisten SM, Weers-Pothoff G, et al. Specific and sensitive detection of Neisseria gonorrhoeae in clinical specimens by real-time PCR. J Clin Microbiol. 2005;43(11):5653-9.

5. Whiley DM, Buda PJ, Bayliss J, Cover L, Bates J, Sloots TP. A new confirmatory Neisseria gonorrhoeae real-time PCR assay targeting the porA pseudogene. Eur J Clin Microbiol Infect Dis. 2004;23(9):705-10.

6. Hjelmevoll SO, Olsen ME, Sollid JU, Haaheim H, Unemo $\mathrm{M}$, Skogen V. A fast real-time polymerase chain reaction method for sensitive and specific detection of the Neisseria gonorrhoeae porA pseudogene. J Mol Diagn. 2006;8(5):574-81. 
7. Whiley DM, Anderson TP, Barratt K, Beaman MH, Buda PJ, Carter M, et al. Evidence that the gonococcal porA pseudogene is present in a broad range of Neisseria gonorrhoeae strains; suitability as a diagnostic target. Pathology. 2006;38(5):445-8.

8. Goire N, Nissen MD, LeCornec GM, Sloots TP, Whiley DM. A duplex Neisseria gonorrhoeae real-time polymerase chain reaction assay targeting the gonococcal porA pseudogene and multicopy opa genes. Diagn Microbiol Infect Dis. 2008;61(1):6-12.

9. Limnios EA, Nguyen NL, Ray S, Mclver CJ, Tapsall JW. Dynamics of appearance and expansion of a prolyliminopeptidasenegative subtype among Neisseria gonorrhoeae isolates collected in Sydney, Australia, from 2002 to 2005. I Clin Microbiol. 2006;44(4):1400-4.

10. The CDS Antibiotic Susceptibility Test. [Accessed 8 Feb 2011] Available from: http://web.med.unsw.edu.au/cdstest

11. Bennett JS, Jolley KA, Sparling PF, Saunders NJ, Hart CA, Feavers IM, et al. Species status of Neisseria gonorrhoeae: evolutionary and epidemiological inferences from multilocus sequence typing. BMC Biol. 2007;5:35.

12. Whiley DM, Goire N, Lambert SB, Nissen MD, Sloots TP, Tapsall JW. Reduced susceptibility to ceftriaxone in Neisseria gonorrhoeae is spread internationally by genetically distinct gonococcal populations. J Antimicrob Chemother. 2011;66(5):1186-7. 